poly(A) is added at the nuclear stage. Similar questions to those asked about the nuclear addition of poly(A) can be directed to the cytoplasmic additionare only certain messengers selected for polyadenylation, what happens to messengers which do not receive poly(A), does polyadenylation have some role in controlling translation? No function for poly $(A)$ in the cytoplasm is yet apparent, but with teleological arguments becoming ever more popular in molecular and cell biology, many will be tempted to conclude that some important function of the poly $(A)$ remains to be discovered.

\section{Eliminating Doppler broadening}

from a Correspondent

THE spectra of atoms (and molecules) are replete with information some of which is unfortunately inaccessible because the fine structure of spectral lines is often unresolved. Consequently, spectroscopists strive constantly to improve their resolution. The limitations imposed on spectral resolution are not, however, always instrumental but can be inherent in the atomic assembly. For example, atoms of a gas present a whole range of velocities to the direction of propagation of a light source because of their thermal motions. Now if $\nu_{0}$ is the frequency of radiation required to raise an atom from its (sharp) lower state to a (sharp) upper energy state when it is stationary relative to the source, then an atom receding from the source 'sees' a number of waves per second (that is a frequency) less than $\nu_{0}$. The receding atom must, of course, absorb radiation which it perceives to have frequency $\nu_{0}$ and which relative to the stationary source must therefore exceed $\nu_{0}$. That the Maxwell-Boltzmann distribution of atomic velocities in the source direction ensures a distribution of absorption frequencies even though each atom has sharp energy levels-the so-called Doppler broadening.

Doppler broadening can be overcome if one selects only atoms within a narrow band of velocities relative to the source so that they all absorb at the same frequency. Several velocity selection techniques are used, including atomic beams and laser saturation spectroscopy (see Nature, 235, 127; 1972). A further, elegant method of dealing with Doppler broadening is now described independently by two groups (Biraben, Cagnac and Grynberg, Phys. Rev. Lett., 23, 643; 1974; Levenson and Bloembergen, ibid., 645).

The essence of the technique used by these authors is very simple. Both groups investigate the normally forbidden $5 S \leftarrow 3 S$ electronic transition of the sodium atom by means of two- photon absorption. This transition is, in fact, a closely-spaced doublet (because of coupling of electronic and nuclear spin angular momentum) with separation $784 \mathrm{MHz}$ but its resolution is precluded in the presence of the aforementioned Doppler broadening which confers a half-width of about $1,000 \mathrm{MHz}$ on each component.

Low pressure sodium vapour is subjected to monochromatic radiation from a tunable laser at a frequency equal to one half that of the $5 \mathrm{~S} \leftarrow 3 \mathrm{~S}$ transition but corresponding to no other known energy state separation within the atom. Those few sodium atoms of thermal velocity such that the $5 S \leftarrow 3 S$ level separation is Dopplershifted into resonance with twice the laser frequency can absorb simultaneously (but with low probability) two photons from the radiation and so raise atoms to the $5 \mathrm{~S}$ state. As the laser is tuned, a weak Doppler broadened absorption profile (detected by counting photons emitted from the $5 \mathrm{~S}$ state) is generated as shown in the figure $(a)$. When, however, a mirror is positioned so as to reflect the radiation back through the sodium vapour and establish within it a radiation standing wave composed of two oppositely travelling waves, a given atom can absorb two photons by an additional mechanismone from each of the counterpropagating beams.

It is clear therefore that for any atom the Doppler shift associated with absorption of a photon from one beam is equal and opposite to that associated with absorption from the other beam. The net result is that each and every atom, regardless of its velocity relative to the radiation source, absorbs two photons from the standing wave but only when the laser frequency is exactly one half the $5 S \leftarrow 3 S$ level separation. When the laser is tuned through this frequency the doublet character of the transition is resolved (see figure $(b)$ ), the Doppler broadening having been neatly eliminated.

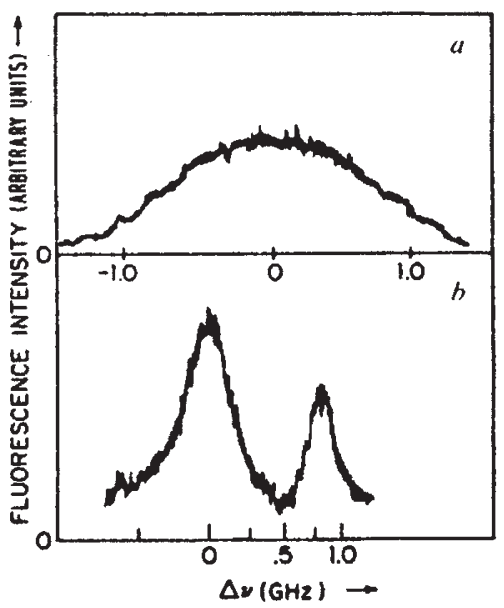

One expects the weak Doppler broadened signal already mentioned as arising from absorption of two photons from one of the component travelling waves to provide a background pedestal to the observed doublet of figure $(b)$, but this has been eliminated by an additional sophistication of the experiment. The two oppositely travelling beams are arranged to be circularly polarised in opposite senses. A rigorous selection rule governing transitions between atomic electron states precludes the $5 \mathrm{~S} \leftarrow 3 \mathrm{~S}$ transition if both photons are absorbed from a single beam of radiation circularly polarised in only one sense but not if one photon is absorbed from each of two beams of opposite circular polarisation. The weak Doppler broadened pedestal is therefore excluded while the sharp doublet remains.

\section{Little evidence for Palaeozoic arthropod and plant interaction}

from Barry Cox

Vertebrate Palaeontology Correspondent

ON April 30, the Palaeobotanical Group of the Linnean Society of London organised a symposium on the interrelationships of Palaeozoic terrestrial arthropods and plants, which took place at the society's rooms in Burlington House. Several contributions set the Palaeozoic scene. M. A. Calver (University of Leeds) discussed the depositional environment of the British Coal Measures. $\mathrm{He}$ showed the gradually decreasing frequency of the marine incursions into this lowlying area of coastal swamps, and briefly reviewed the two conflicting theories of the deposition of coal. R. H. Wagner (University of Sheffield) described the distribution of the Upper Carboniferous flora of the world. $\mathrm{He}$ contrasted the rich equatorial flora of Euramerica with the poorer and more conservative Gondwana flora, and showed that there were some indications of different swampland, dryland and upland floras.

Turning to the vertebrates, A. L. Panchen (University of Newcastle upon Tyne) described the ecology and distribution of Carboniferous tetrapods, and suggested that the tetrapods might have evolved in Euramerica. Though herbivorous reptiles probably evolved in the Upper Carboniferous, their remains are rare until the Lower Permian.

The general evolutionary background of the arthropod radiation was described by S. M. Manton (Queen Mary College, London). W. D. I. Rolfe (University of Glasgow) dealt with the 